\title{
Detection of residual rifampicin in urine via fluorescence quenching of gold nanoclusters on paper
}

\author{
Krishnendu Chatterjee ${ }^{1,2,3,4}$, Chiung Wen Kuo ${ }^{4^{*}}$, Ann Chen $^{5}$ and Peilin Chen ${ }^{*}$
}

\begin{abstract}
Background: Rifampicin or rifampin (R) is a common drug used to treat inactive meningitis, cholestatic pruritus and tuberculosis (TB), and it is generally prescribed for long-term administration under regulated dosages. Constant monitoring of rifampicin is important for controlling the side effects and preventing overdose caused by chronic medication. In this study, we present an easy to use, effective and less costly method for detecting residual rifampicin in urine samples using protein (bovine serum albumin, BSA)-stabilized gold nanoclusters (BSA-Au NCs) adsorbed on a paper substrate in which the concentration of rifampicin in urine can be detected via fluorescence quenching. The intensity of the colorimetric assay performed on the paper-based platforms can be easily captured using a digital camera and subsequently analyzed.

Results: The decreased fluorescence intensity of BSA-Au NCs in the presence of rifampicin allows for the sensitive detection of rifampicin in a range from 0.5 to $823 \mu \mathrm{g} / \mathrm{mL}$. The detection limit for rifampicin was measured as $70 \mathrm{ng} /$ $\mathrm{mL}$. The BSA-Au NCs were immobilized on a wax-printed paper-based platform and used to conduct real-time monitoring of rifampicin in urine.

Conclusion: We have developed a robust, cost-effective, and portable point-of-care medical diagnostic platform for the detection of rifampicin in urine based on the ability of rifampicin to quench the fluorescence of immobilized BSAAu NCs on wax-printed papers. The paper-based assay can be further used for the detection of other specific analytes via surface modification of the BSA in BSA-Au NCs and offers a useful tool for monitoring other diseases.
\end{abstract}

Keywords: Fluorescent biosensors, Fluorescence quenching, Gold nanocluster, Rifampicin detection, TB drug monitoring, Wax-printed paper platform

\section{Background}

Rifampicin is an important bactericidal antibiotic drug of the rifamycin group, which inhibits DNA-dependent RNA polymerase and suspends bacterial growth. This drug is widely used to target Bacillus and Listeria strains and commonly prescribed together with other drugs for the treatment of tuberculosis (TB) [1-3] and infections associated with osteomyelitis, prosthetic joints, and meningitis. In the treatment of TB, three frontline drugs, isoniazid (INZ), ethambutol (E) and pyrazinamide (P),

\footnotetext{
*Correspondence: kuo55@gate.sinica.edu.tw; peilin@gate.sinica.edu.tw

${ }^{4}$ Research Center for Applied Sciences, Academia Sinica, Taipei 115,

Taiwan, ROC

Full list of author information is available at the end of the article
}

are given together with rifampicin. In a standard treatment procedure for $\mathrm{TB}$, all four drugs are administered in various combinations over the first 2 months, and isoniazid and rifampicin are continued for the next 4 months [1]. Improper dosage during the sustained medication period (2-6 months) often leads to drug resistance and even death despite the diseases being curable. Therefore, it is necessary to monitor possible irregularities in drug dosage. The simplest approach for detecting irregularity in drug dosage is to assess the residual rifampicin in the urine of patients; however, inaccurate results could adversely affect the patient and lead to an increased risk of hepatotoxicity because of increased ingestion and drug accumulation in the liver. Thus, various methods 
have been applied to detect rifampicin levels, including spectrophotometry [4], chemiluminescence [5], electrochemistry [6], and high-performance liquid chromatography (HPLC) [7-11]. However, it is desirable to develop an alternative approach for on-site detection of residual rifampicin in urine. Recently, a paper-based sensing platform was demonstrated as an attractive alternative to conventional analytical instrumentation for point-of-care medical diagnostics $[12,13]$, and it is ideal for the detection of residual rifampicin in urine because of its advantages, including its low cost, quick response time, ease of handling and disposability. However, the selectivity and sensitivity of paper-based sensors are highly dependent on the sensing scheme.

With recent developments in nanotechnology, nanoparticle-based sensors have demonstrated notably high sensitivity in various applications [14-19]. Fluorescent metal nanoclusters (NCs) [20-27] are a recent addition to a growing list of labeling and sensing agents that have generated massive interest among researchers because of their biocompatibility, non-toxicity and outstanding fluorescent properties. Fluorescent gold nanoclusters manufactured via eco-friendly one-pot synthesis approaches using commonly available templating agents (i.e., bovine serum albumin (BSA) [20], lysozyme [21], rec1-resilin [22], transferrin [23], glutathione [24], insulin fibrils [25], horseradish peroxidase [26] and papain [27]) have been reported by several groups. Among these materials, BSAstabilized metal nanoclusters have been widely used as fluorescent turn-off probes for the detection of methotrexate (MTX) [28], quercetin [29], cyanide [30], glucose [31], $\mathrm{Hg}^{2+}$ [32], and $\mathrm{Cu}^{2+}$ in aqueous solution [33] and even $\mathrm{Cu}^{2+}$ in live cells [34]. To date, various NC applications have been reported as fluorescent probes used in the solution phase [28-34]. However, few studies [35] have explored the immobilization of NCs on paper and their subsequent use as a sensing platform. Based on the concept of paper chromatography, immobilized paper sensors have been used in modern laboratories for a number of years. However, applications with paper-based drug monitoring devices have not been fully explored.

In this experiment, a commercially available printer and hot plate were used to create multi-zone standard 96-well paper microplates as an alternative to conventional polymer multiwell plates [12, 13]. The fluorescent BSA-Au NCs were immobilized on a wax-printed paper platform in which the fluorescence intensity from the immobilized BSA-Au NC was quenched in the presence of rifampicin. Rifampicin exhibited a greater binding affinity with BSA than isoniazid, and the binding order of rifampicin with BSA is approximately $10^{4} \mathrm{~L} / \mathrm{mol}$ [36], whereas it is approximately $10^{3} \mathrm{~L} / \mathrm{mol}$ [37] with isoniazid. Therefore, the BSA-Au NC-modified paper sensor can be used to detect the residual rifampicin in urine. Figure 1 presents a schematic of the preparation and detection strategy of the paper sensor for residual rifampicin in urine. The BSA-Au NC microplate paper sensor can be fabricated in three steps: printing patterns on the paper,

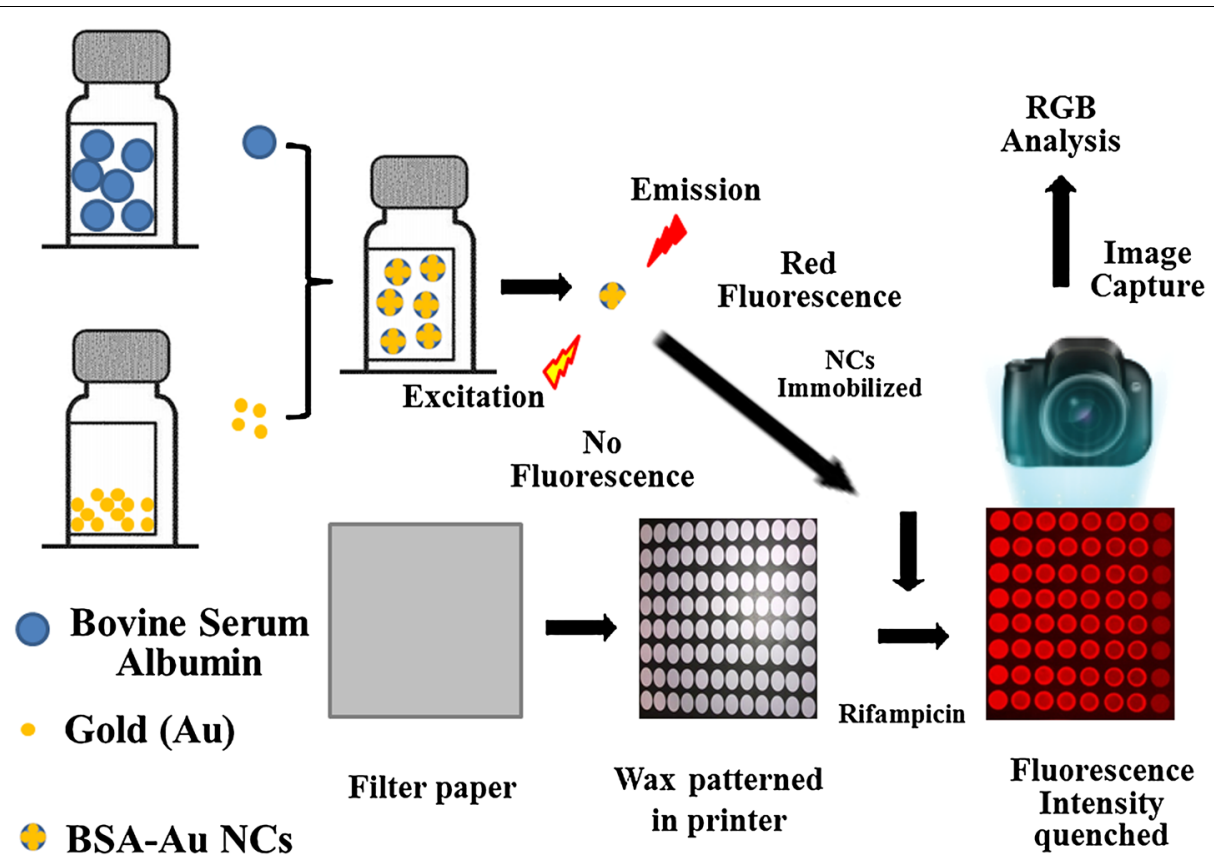

Figure 1 Schematic illustration of the synthesis, BSA-Au NC immobilization on paper and application to detect rifampicin. 
melting wax to form hydrophobic barriers, and immobilizing the BSA-Au NCs in the reaction zones. After dropping the samples onto the paper sensor, the fluorescence images are captured by a digital camera and analyzed [38-40].

\section{Results and discussion Characterization of BSA-Au NCs}

The BSA-Au NCs were developed using alkaline coprecipitation of gold salt with protein BSA [41], and they exhibited a sharp and intense emission peak centered at approximately $640 \mathrm{~nm}$ when excited at $480 \mathrm{~nm}$ (Additional file 1: Figure S1). The emission band centered at $640 \mathrm{~nm}$ began to appear after heating the reactants for approximately $10 \mathrm{~min}$, and the emission intensity increased proportionally with $30 \mathrm{~min}$ of heating, although upon further heating, the intensity gradually decreased. Therefore, the optimal reaction time was $30 \mathrm{~min}$ for the synthesis of fluorescent BSA-Au NCs at $70^{\circ} \mathrm{C}$ at a $\mathrm{pH}$ of 11.5 (Additional file 1: Figure S1). The maximum fluorescence intensity of the emission peak of the BSA-Au NCs was obtained at an excitation wavelength of $480 \mathrm{~nm}$ (Additional file 1: Figure S2). Thus, the selection of $480 \mathrm{~nm}$ as the excitation wavelength with an emission peak centered at $640 \mathrm{~nm}$ and large Stokes shift (greater than $150 \mathrm{~nm}$ ) eliminated the possibility of fluorescence self-quenching and measurement errors that might be caused by excitation light and scattering. The quantum yield of the prepared BSA-Au NCs was approximately $2 \%$ compared with that of Rhodamine $6 \mathrm{G}$. In addition, the prepared BSA-Au NCs showed excellent stability over a wide range of $\mathrm{pH}$ values (2.0-10.0) (Additional file 1: Figure S3).

Solid-state Fourier transform infrared spectroscopy (FTIR) spectra were analyzed to investigate the surface structural changes after conjugating Au with BSA. The FTIR spectrum (Additional file 1: Figure S4) confirmed the presence of amide A, amide I, and amide II bands at $3,302.09 \mathrm{~cm}^{-1}, 1,661.14 \mathrm{~cm}^{-1}$ and $1,530.14 \mathrm{~cm}^{-1}$, respectively, in pure BSA. The spectra of the BSA-Au $\mathrm{NCs}$ also showed three prominent bands corresponding to amide A, amide I, and amide II of the dipeptide at $3,297.41 \mathrm{~cm}^{-1}, 1,658.18 \mathrm{~cm}^{-1}$ and $1,531.34 \mathrm{~cm}^{-1}$, respectively, which clearly demonstrated the dipeptide presence on the Au cluster surfaces. Additionally, a band at $2,961.20 \mathrm{~cm}^{-1}$ corresponding to $\mathrm{C}-\mathrm{H}$ vibrations modes observed in pure BSA can also be observed in BSA-Au NCs. After adding rifampicin to the NCs, the FTIR spectra showed a considerable decrease in the broad band centered around 3,293.01. Although the characteristic peak of BSA at 3,293.01, 1,645.43 and 1,535.64 $\mathrm{cm}^{-1}$ were quite evident in the spectra (BSA-Au+R); peak broadening after the addition of rifampicin to the BSA-Au NCs could point towards the formation of hydrogen bonding between rifampicin and the BSA-Au NCs. The FTIR analysis illustrated that the surface structure of BSA remained intact even after the formation of BSA-Au $\mathrm{NCs}$, implying that the BSA retained its reactive sites on the surface and could be further modified with new functional groups.

The size and shape of the BSA-Au NCs were investigated via electron microscopy. Additional file 1: Figure S5 presents the scanning transmission electron microscopy (STEM) (a) and high-resolution transmission electron microscopy (HRTEM) (b) images of the prepared BSA$\mathrm{Au}$ NCs. The STEM and HRTEM images indicate that the NCs are well dispersed and the average diameter of the BSA-Au NCs is approximately $1.1 \pm 0.1 \mathrm{~nm}$ as calculated from the images via ImageJ (Additional file 1: Figure S6).

Matrix-assisted lasers desorption/ionization mass spectroscopy (MALDI-MS) analysis was performed to reveal the number of $\mathrm{Au}$ atoms in the BSA-Au NCs. The samples were dialyzed against water using a $10-\mathrm{kDa}$ cutoff dialysis bag prior to MALDI-MS analysis. A broad peak for pure BSA was observed at $m / z 65.4 \mathrm{kDa}$ (Additional file 1: Figure S7a). After the reaction time was increased, the peaks for BSA-Au NCs became broader, and at a 30-min reaction time, we estimated that 18-atom $\mathrm{Au}$ NCs were formed $(m / z$ 68.9) (Additional file 1: Figure $\mathrm{S} 7 \mathrm{~b})$. The BSA undergoes partial denaturation upon heating at $70^{\circ} \mathrm{C}$ to expose amino residues, including histidine, cysteine, and tyrosine, which interact more strongly with $\mathrm{Au}^{+}\left(\mathrm{Au}^{3+}\right)$ to form NCs. Thus, one BSA conjugates with an 18-atom Au in the core to form BSA-Au NCs. The size of the formed $\mathrm{Au}$ cores is much smaller than that of BSA$\mathrm{Au}_{25} \mathrm{NCs}$ prepared at room temperature for $12 \mathrm{~h}$ [20] because of the higher energy provided for the formation of BSA-Au NCs. On addition of $10 \mathrm{mM}$ rifampicin to the BSA-stabilized $\mathrm{Au} \mathrm{NC}$, the MS spectra indicate that the characteristic peak of the BSA-Au NCs remained intact, thus indicating that leaching of $\mathrm{Au}$ had not occurred from the core of the BSA-Au NCs (Additional file 1: Figure S7c).

\section{Quenching mechanism of BSA-Au NCs by rifampicin}

After adding rifampicin, the emission intensity of the $\mathrm{NCs}$ was greatly reduced. The MALDI-MS spectra (Additional file 1: Figure S7) of BSA-Au NCs both before and after adding rifampicin were quite similar, thus indicating the absence of any leaching of Au from the core of the BSA-Au NCs. To further prove that Au is not leached from the core of the BSA-Au NCs, an inductively coupled plasma mass spectrometry (ICP-MS) (data not shown) analysis was performed to determine the $\mathrm{Au}$ content of the purified BSA-Au NCs both in the presence and 
absence of rifampicin. The prepared aqueous solution of BSA-Au NCs had an Au content of $404.4 \mu \mathrm{g} / \mathrm{mL}$, whereas upon interaction with rifampicin, $392.4 \mu \mathrm{g} / \mathrm{mL}$ of Au was detected in the aqueous solution of BSA-Au, revealing that the $\mathrm{Au}$ atoms in the NCs remained intact and were not filtered during dialysis.

$\mathrm{Au}$ is conjugated with BSA through covalent bonding between the Au and thiol groups of the cysteine in BSA [41]. Rifampicin does not substitute the BSA and drive $\mathrm{Au}$ NC aggregation, which is evident from the MALDIMS and ICP-MS data. Moreover, the absorption spectra indicate that aggregation did not occur, even after a long period of time following the addition of rifampicin to BSA-Au NCs (Figure 2a). However, the interaction of BSA and rifampicin has been previously reported [36]. Therefore, BSA-Au NC quenching by rifampicin (Figure $2 \mathrm{~b}$ ) may have been caused by the interaction of BSA and rifampicin, which changes the environment of the $\mathrm{Au}$ NCs. Changes in the absorption spectra of BSA-Au $\mathrm{NCs}$ in the presence of rifampicin (shown in Figure 2a) are similar to those reported for the interaction of BSA and rifampicin alone [36]. The absence of aggregated $\mathrm{Au}$ NCs without BSA protection corroborates the formation of structures similar to the rifampicin-RS-Au bonds.

Particle size measurements might offer a more sensitive tool for studying nanoparticle aggregation and hydrodynamic size distributions. BSA is a $66 \mathrm{kDa}$ protein with a diameter of approximately 7-8 nm (data from Protein Data Bank). The dynamic light scattering (DLS) analysis could detect and measure the protein size directly (Additional file 1: Figure S8). The hydrodynamic size of BSA increases upon NC formation which can be conveniently observed via DLS. The diameter of the BSA-Au NCs increases to at least two times the diameter of the protein (BSA) molecule. After adding rifampicin to the NC, the hydrodynamic size of the BSA-Au NCs further increased significantly (Additional file 1: Figure S8), indicating the bonding of rifampicin to the surface of the NC.

\section{Fluorescence detection of rifampicin}

With the inherent fluorescence of BSA-Au NCs and its high sensitivity, the synthesized NCs could be used to detect rifampicin via rapid fluorescence quenching. The fluorescence intensity of the BSA-Au NCs decreased proportionally with increases in rifampicin concentration (Additional file 1: Figure S9). As shown in Figure 3a, a nearly linear relationship was observed between the normalized decrease in fluorescence intensity $\left(\mathrm{F}_{0}-\mathrm{F}\right) / \mathrm{F}$ and concentration of rifampicin over a range from 0.5 to $823 \mu \mathrm{g} / \mathrm{mL}$. However, the normalized decrease in fluorescence intensity reached a plateau when the concentration of rifampicin fell below $0.5 \mu \mathrm{g} / \mathrm{mL}$.

Plotting of the normalized decrease in fluorescence intensity $\left(\mathrm{F}_{0}-\mathrm{F}\right) / \mathrm{F}$ versus the $\log$ of the rifampicin concentration resulted in a linear calibration graph over the concentration range from 0.5 to $823 \mu \mathrm{g} / \mathrm{mL}$ (correlation coefficient: $\left.\mathrm{R}^{2}=0.9945\right)$ with a limit of detection (LOD) of $0.07 \mu \mathrm{g} / \mathrm{mL}$ (Figure 3b). The limit of quantification (LOQ) was $0.2 \mu \mathrm{g} / \mathrm{mL}$. The reproducibility of the sensing system was established by performing three independent measurements using $20.6 \mu \mathrm{g} / \mathrm{mL}$ rifampicin with a relative standard deviation of $4.22 \%\left(\mathrm{R}^{2}=0.9948\right)$. This measurement was conducted to verify the reliability of the proposed method, which presents high sensitivity and notably low relative standard deviations.

\section{Selectivity of BSA-Au NCs toward detecting rifampicin}

We tested the selectivity of BSA-Au NCs for sensing rifampicin in the presence of other antibiotics (INZ, E and $\mathrm{P}$ ) used collaterally with rifampicin for the treatment
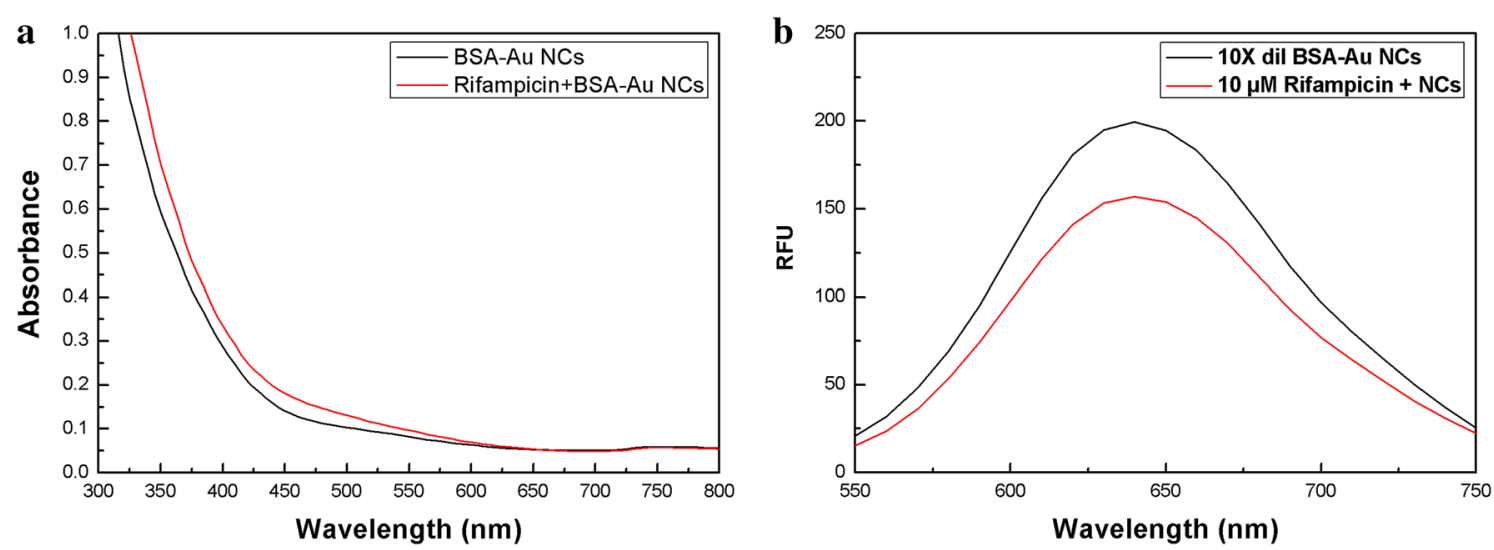

Figure 2 a Absorption spectra of BSA-Au NCs (0.1× dilution) in the absence and presence of $10 \mu \mathrm{M}$ rifampicin. b Fluorescence emission spectra (excitation wavelength at $480 \mathrm{~nm})$ of BSA-Au NCs $(0.1 \times$ dilution) in the absence and presence of $10 \mu \mathrm{M}$ rifampicin. 

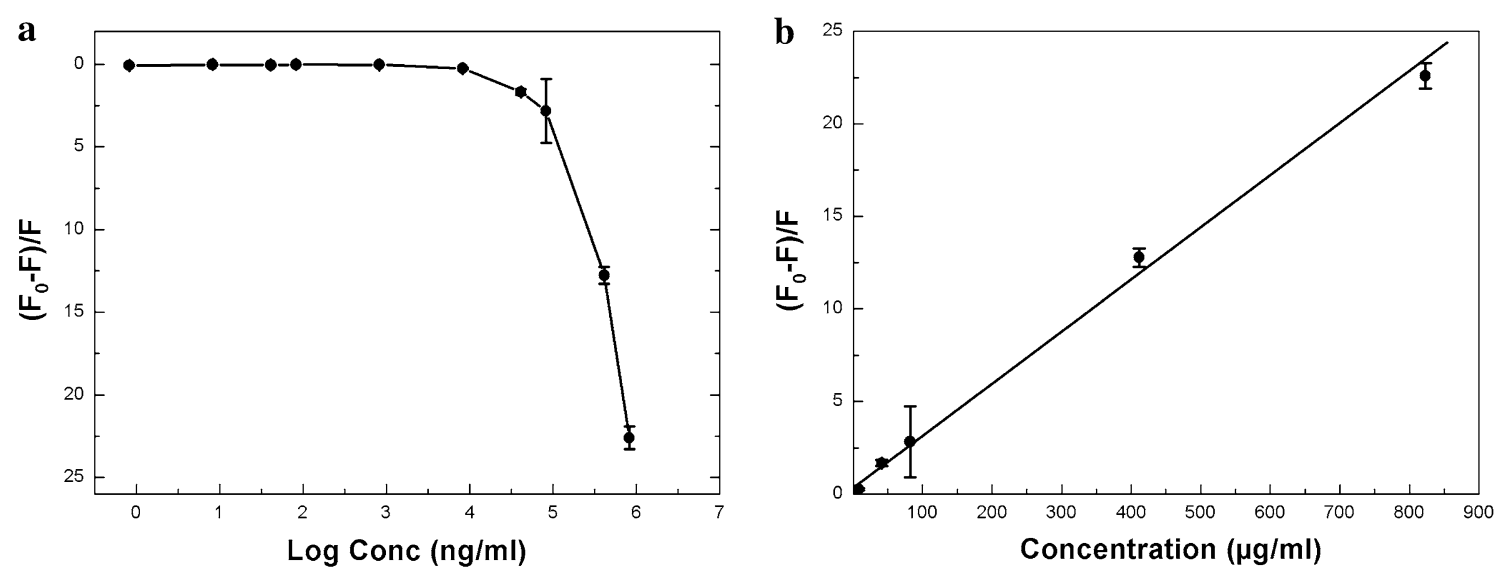

Figure 3 a Concentration-dependent quenching of BSA-Au NCs ( $0.1 \times$ dilution) by rifampicin. From higher to lower concentrations: normalized quenching at $823 \mu \mathrm{g} / \mathrm{mL}\left[\left(F_{0}-\mathrm{F}\right) / \mathrm{F} \sim 22.6 \pm 0.68\right], 411 \mu \mathrm{g} / \mathrm{mL}[12.78 \pm 0.5], 82 \mu \mathrm{g} / \mathrm{mL}[2.8 \pm 1.93], 41 \mu \mathrm{g} / \mathrm{mL}[1.68 \pm 0.16], 8 \mu \mathrm{g} / \mathrm{mL}[0.25 \pm 0.6]$, $4 \mu \mathrm{g} / \mathrm{mL}[0.23 \pm 0.008], 0.8 \mu \mathrm{g} / \mathrm{mL}[0.016 \pm 0.009], 0.4 \mu \mathrm{g} / \mathrm{mL}[0.046 \pm 0.031], 0.08 \mu \mathrm{g} / \mathrm{mL}[0.017 \pm 0.016]$, and $0.004 \mu \mathrm{g} / \mathrm{mL}$ [0.06 \pm 0.04$]$ (each data point represents the average of three separate studies $(n=3)$, and the error bars denote the standard error of measurements within each experiment). $\mathbf{b}$ Plot of the linear region of the normalized decrease in fluorescence intensity of BSA-Au NCs ( $0.1 \times$ dilution) versus rifampicin concentration (each data point represents an average of three separate studies $(n=3)$; the error bars denote the standard error of measurements within each experiment). The excitation wavelength was set at $480 \mathrm{~nm}$, and the emission wavelength was $640 \mathrm{~nm}$.

of TB. The fluorescent responses of BSA-Au NCs with all four antibiotics were monitored and compared with that of rifampicin. Figure 4a shows that the addition of rifampicin at a final concentration of $100 \mu \mathrm{M}(83 \mu \mathrm{g} /$ $\mathrm{mL}$ ) resulted in a nearly $90 \%$ decrease in the fluorescence intensity of the BSA-Au NCs, whereas a limited decrease in fluorescence intensity was observed for $\mathrm{P}(1.3 \%)$, INZ $(2.8 \%)$ and $\mathrm{E}(6.5 \%)$ at the same molar concentration (Figure $4 \mathrm{~b}$ ). The clinical effectiveness of rifampicin as a primary drug for TB can mainly be attributed to 4-methyl-1-piperazinaminyl substitution on rifampicin. Generally, antibiotics with piperazine moiety exhibit
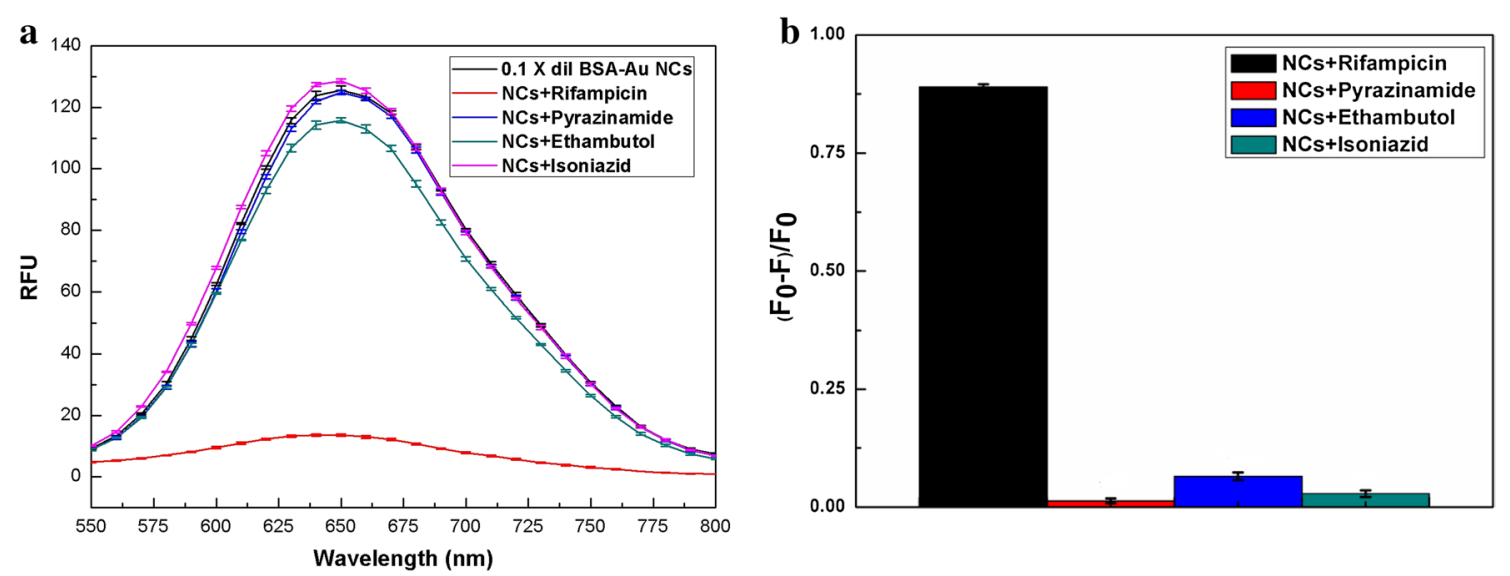

Figure 4 a Fluorescence emission spectra of BSA-Au NCs $(0.1 \times$ dilution $)$ in the presence of primary TB drugs $(100 \mu \mathrm{M}$ each in the final concentration). At $640 \mathrm{~nm}$ (emission wavelength): BSA-Au NCs (fluorescence intensity $=123.74 \pm 1.56), \mathrm{NCs}+$ rifampicin $(83 \mu \mathrm{g} / \mathrm{mL})(13.67 \pm 0.33)$, $\mathrm{NCs}+$ pyrazinamide $(12.3 \mu \mathrm{g} / \mathrm{mL})(121.95 \pm 0.69), \mathrm{NCs}+$ ethambutol $(27.7 \mu \mathrm{g} / \mathrm{mL})(114.27 \pm 1.28)$ and NCs + izoniazid $(13.7 \mu \mathrm{g} / \mathrm{mL})(127 \pm 0.69)$ (each data point represents the average of three separate studies $(n=3)$, and the error bars denote the standard error of measurements within each experiment). $\mathbf{b}$ Comparison of the normalized decrease in fluorescence intensity of BSA-Au NCs $(0.1 \times$ dilution) in the presence of primary TB drugs (100 $\mu \mathrm{M}$ each in final concentration). At $640 \mathrm{~nm}$ (emission wavelength): normalized quenching of $\mathrm{NCs}+$ rifampicin $\left[\left(\mathrm{F}_{0}-\mathrm{F}\right) / \mathrm{F}_{0} \sim 0.89 \pm 0.006\right]$, $\mathrm{NCS}+$ pyrazinamide $[0.013 \pm 0.005], \mathrm{NCS}+$ ethambutol $[0.065 \pm 0.008]$ and NCs + izoniazid [0.028 \pm 0.007$]$ (each data point represents the average of three separate studies $(n=3)$, and the error bars denote the standard error of measurements within each experiment). [ $\left(n\right.$ this work, $\left(F_{0}-F\right) /$ $\mathrm{F}_{0}=1$ indicates complete quenching and $\left(\mathrm{F}_{0}-\mathrm{F}\right) / \mathrm{F}_{0}=0$ indicates no quenching]. The excitation wavelength was set at $480 \mathrm{~nm}$, and the emission wavelength was $640 \mathrm{~nm}$. 
good efficacy as therapeutic agents [42-44]. Therefore, we tested the specificity of BSA-Au NCs toward detecting rifampicin using antibiotics with piperazine functional group such as ciprofloxacin [42], buspirone [43], ipsapirone [44] (Additional file 1: Figure S10a). In addition, we also measured the fluorescence responses of some commonly used antibiotics and drugs (Additional file 1: Figure S10b). The average amount of residual rifampicin present in the patients' urine is in the range of $45-55 \mu \mathrm{g} /$ $\mathrm{mL}$ [45]. Our results showed that at a final concentration of $50 \mu \mathrm{g} / \mathrm{mL}$ of rifampicin a nearly $60 \%$ decrease in the fluorescence intensity of the BSA-Au NCs was measured, whereas only a limited decrease in fluorescence intensity (less than 10\%, p < 0.001) was observed for all the other antibiotics and drugs at the same concentration. Therefore, BSA-Au NCs can be used for preferential sensing of rifampicin among other antibiotics administered at the same time. Additionally, the prepared BSA-Au NCs were freeze-dried and stored for 3 months before dispersion for the same experiment (Additional file 1: Figure S11). No drastic changes were observed, even after storage, indicating the excellent stability of the BSA-Au NC.

\section{Tolerance to potentially interfering ions}

In a real urine sample, the presence of ions might interfere with rifampicin sensing. To evaluate the tolerance level to these ions, solutions containing specific ions were tested until the interfering substances had a minimal effect on fluorescence quenching of the BSA-Au NCs (less than 5\% change). The results from the potentially interfering substances are presented in the Additional file 1: Table S1 together with the typical ion concentrations found in the real urine sample. The selective interaction of BSA-rifampicin was stronger than other ionic interferences. At a tenfold dilution, the interference from ions in urine is negligible.

\section{Detection of rifampicin in spiked urine}

After determining that the BSA-Au NCs have excellent sensitivity and specificity in sensing rifampicin, the next step was to determine the concentration of rifampicin in urine using BSA-Au NCs. Generally, evaluating the intake dosage of rifampicin for a patient requires urine collection over a long period of time $(8-24 \mathrm{~h})$ after ingestion of the drug followed by analysis in a laboratory equipped with sophisticated instrumentation. In the urine sample, the average indicative levels of rifampicin intake ranged from 45 to $55 \mu \mathrm{g} / \mathrm{mL}$ [45]. In our experiment, various concentrations of rifampicin were spiked into $10 \mathrm{~mL}$ of fresh urine collected from a healthy individual. A tenfold dilution of the urine sample containing rifampicin was used for the quantitative analysis via fluorescence quenching (turn-off sensing mechanism). The recovery of rifampicin was estimated at greater than $85 \%$ in the concentration range normally present in actual urine samples (i.e., 5-60 $\mu \mathrm{g} / \mathrm{mL}$ ). Additional file 1: Table S2 presents the comparative results for determining rifampicin levels using other assays.

\section{BSA-Au NCs immobilized on a paper platform for detection of rifampicin in spiked urine}

Disposable sensors are desirable in point-of-care applications. Therefore, we immobilized BSA-Au NCs on a wax-printed paper-based platform to conduct real-time monitoring of rifampicin in urine. The urine samples were collected from patients at the Tri-Service General Hospital in Taipei, Taiwan. The patients who contributed urine samples signed informed consent forms as required by the regulations of the Institutional Review Board of the Tri-Service General Hospital of Taipei, Taiwan. Initially, $30 \mu \mathrm{L}$ of the prepared BSA-Au NCs were dropped in each well of the 96-well wax-printed paper platform and dried. The changes observed after the BSA-Au NCs were immobilized on the paper under ultraviolet (UV) light are shown in Figure $5 \mathrm{a}(\mathrm{A})$. The images were captured using a digital camera (Olympus, E-330), and the images were processed by MetaMorph software using only the 'red' color and center portion of each well to calculate the effective fluorescence intensity quenching ratios. The area without BSA-Au NCs (blank) was used as the background. Pale red fluorescence was clearly observed after immobilization of the BSA-Au NCs on the paper platform. Different amounts of rifampicin were spiked in the collected urine samples, and tenfold diluted urine samples were used for the quantitative analysis.

Increases in rifampicin concentration increased the fluorescence quenching of the imbedded BSA-Au NCs in the wax-printed paper sensor. Each colorimetric assay was performed using a total volume of $30 \mu \mathrm{L}$ prepared BSA-Au NCs, and the evaluation was performed with a total of $30 \mu \mathrm{L}$ spiked urine containing different concentrations of rifampicin. The amount of fluorescence quenching was lowest with spiked urine containing $0.5 \mu \mathrm{g} / \mathrm{mL}$ rifampicin (Figure $5 \mathrm{a}(\mathrm{B})$ ), followed by that of $5 \mu \mathrm{g} / \mathrm{mL}$ (Figure $5 \mathrm{a}(\mathrm{C})$ ), $10 \mu \mathrm{g} / \mathrm{mL}$ (Figure $5 \mathrm{a}(\mathrm{D})$ ), $30 \mu \mathrm{g} /$ $\mathrm{mL}$ (Figure $5 \mathrm{a}(\mathrm{E})$ ), $50 \mu \mathrm{g} / \mathrm{mL}$ (Figure $5 \mathrm{a}(\mathrm{F})$ ), $100 \mu \mathrm{g} / \mathrm{mL}$ (Figure $5 \mathrm{a}(\mathrm{G})$ ), $500 \mu \mathrm{g} / \mathrm{mL}$ (Figure $5 \mathrm{a}(\mathrm{H})$ ) and $1000 \mu \mathrm{g} /$ $\mathrm{mL}$ (Figure $5 \mathrm{a}(\mathrm{I})$ ). Our detection scheme can be further simplified via the use of a mobile imaging device that can determine the ratio of BSA-Au NCs quenching by capturing the image intensity and perform analyses through an installed application [38-40]. This process eliminates the need to install an image analyzer at the site of rifampicin detection. Figure $5 \mathrm{~b}$ plots the data obtained from three different paper sensors with three independent data points for each concentration of rifampicin. Our 
$\mathbf{a}$

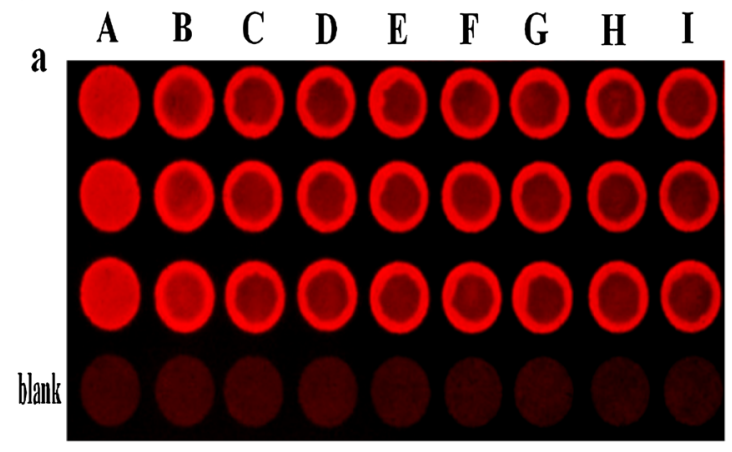

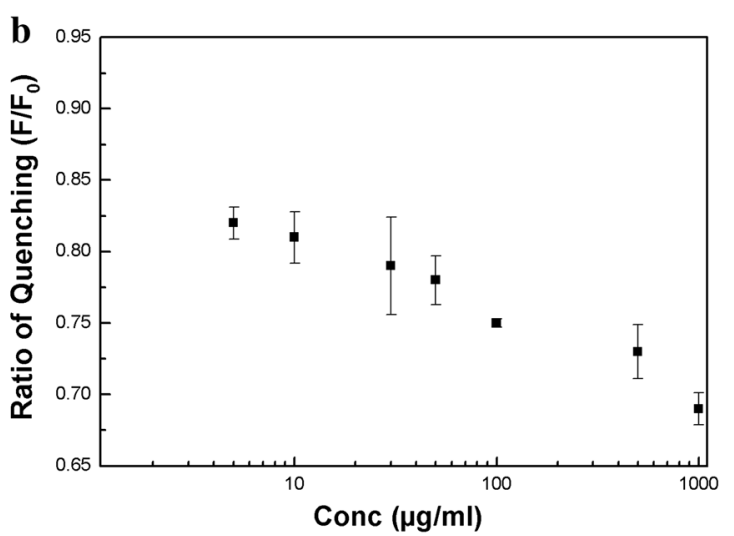

Figure $\mathbf{5}$ a Test paper for the detection of rifampicin after modification (A) with BSA-Au NCs under UV light. The tenfold diluted urine samples with original rifampicin concentrations are as follows: (B) $0.5 \mu \mathrm{g} / \mathrm{mL}$ (fluorescence quenching ratio $=91 \% \pm 1) ;(C) 5 \mu \mathrm{g} / \mathrm{mL}(82 \% \pm 1.1) ;(D)$ $10 \mu \mathrm{g} / \mathrm{mL}$ (81\% \pm 1.8); (E) $30 \mu \mathrm{g} / \mathrm{mL}$ (79\% \pm 3.4); (F) $50 \mu \mathrm{g} / \mathrm{mL}$ (78\% \pm 1.7); (G) $100 \mu \mathrm{g} / \mathrm{mL}$ (75\% \pm 0.3); (H) $500 \mu \mathrm{g} / \mathrm{mL}(73 \% \pm 1.9) ;$ and (I) 1000 $\mu \mathrm{g} /$ $\mathrm{mL}(69 \% \pm 1.1)$. b Change in the fluorescence quenching ratio of the embedded BSA-Au NCs versus rifampicin concentration on the paper sensor (each data point represents an average of three separate studies $(n=3)$ in three different wax-printed 96-microplate paper platforms; three measurements were taken in each micro-well, and the error bars denote the standard deviation of the reading).

results show that even the minimum therapeutic dosage [45] $(5 \mu \mathrm{g} / \mathrm{mL})$ of rifampicin in the urine can be observed with the paper sensor platform. Therefore, the actual amount [45] (45-55 $\mu \mathrm{g} / \mathrm{mL})$ of rifampicin present in the urine sample can be monitored easily using this methodology. The results obtained for all data points were similar when the tests were performed with different paper platforms (Additional file 1: Table S3). This paper-based assay offers numerous advantages over solution-phase fluorescent probes, including its low cost, rapidity, simplicity, robustness and ability to perform real-time monitoring of samples.

\section{Conclusion}

We have developed a robust, cost-effective and portable point-of-care medical diagnostic platform for the detection of rifampicin in urine based on the ability of rifampicin to quench the fluorescence of immobilized $\mathrm{BSA}-\mathrm{Au} \mathrm{NCs}$ on wax-printed paper. Water-soluble Au NCs were synthesized within $30 \mathrm{~min}$ at $70^{\circ} \mathrm{C}$ using BSA as a template. The prepared BSA-Au NCs possessed high fluorescence emission intensity and stability. The BSA-Au NCs showed remarkable selectivity towards rifampicin over other TB drugs. The fluorescent property of the BSA-Au NCs was retained after immobilization on paper, and the quenching of fluorescence by rifampicin in urine can be observed by the naked eye under UV irradiation. We demonstrated that a mobile imaging device can be used in data acquisition to capture the image intensity as well as data analysis to quantify the rifampicin. Therefore, the semi-quantitative assay presented here could be useful for monitoring drug intake in $\mathrm{TB}$ patients on a regular basis and can act as a first line of detection to complement current techniques used to detect rifampicin in urine. The paper-based assay can also be used to detect other specific analytes via surface modification of the BSA in the BSA-Au NCs and has the potential for use in the monitoring of other diseases.

\section{Methods}

Materials

BSA was purchased from Sigma-Aldrich (A7906-10g). Hydrogen tetrachloroaurate(III) trihydrate $\left(\mathrm{HAuCl}_{4}\right)$ and isonicotinic acid hydrazide were obtained from Alfa Aesar. Rifampicin was purchased from Sigma Aldrich (R3501-1g), pyrazinamide was obtained from Acros Organics, and ethambutol dihydrochloride was obtained from LKT Laboratories, Inc. Sodium hydroxide was obtained from Riedel-de Haen. All of the solvents and chemicals were used without further purification. Aqueous solutions were prepared using deionized water (DI) with a resistivity of $18.20 \mathrm{M} \Omega \mathrm{cm}$.

\section{Instrumentation}

A SpectraMax M2 (Molecular Devices) spectrophotometer was used to measure the visible ultraviolet (UV-Vis) absorbance of the protein-Au NCs and their fluorescence intensities with an excitation wavelength of $480 \mathrm{~nm}$ and emission wavelength of $640 \mathrm{~nm}$. The FTIR (PerkinElmer Spectrum 100 FT-IR Spectrometer) spectra were recorded to determine the structural changes before and after conjugation of Au with BSA. The STEM and TEM (JEOL JEM-2100 Field Emission Transmission Electron Microscope) images were 
used to determine the size distributions and measure the dispersibility of the prepared nanoparticles. The MALDI-MS (MALDI TOF-TOF, Bruker, UltraflXtreme) analysis was applied to reveal the number of $\mathrm{Au}$ atoms in the BSA-Au NCs. The ICP-MS (Thermo $\mathrm{X}$-Series II) analysis was applied to determine the $\mathrm{Au}$ content of the purified BSA-Au NCs both in the presence and absence of rifampicin. A 90 Plus particle size analyzer (BIC) was used for the size distribution studies.

\section{Synthesis of BSA-stabilized gold nanoclusters}

According to the reported protocol [41], $5 \mathrm{~mL}$ of an aqueous solution of $\mathrm{HAuCl}_{4}(10 \mathrm{mM})$ was added to $5 \mathrm{~mL}$ BSA ( $50 \mathrm{mg} / \mathrm{mL}$ in water) under vigorous stirring for $30 \mathrm{~s}$. Next, the $\mathrm{pH}$ of the solution was adjusted to approximately 11.5 through the addition of $\mathrm{NaOH}$ $(0.5 \mathrm{~mL}, 1 \mathrm{M})$. The reaction was conducted at $70^{\circ} \mathrm{C}$ and reached its maximum intensity after $30 \mathrm{~min}$ of reaction time with completion of the BSA-Au conjugation (Additional file 1: Figure S1), wherein the solution turned from pale yellow to dark red, thus indicating the formation of BSA-Au NCs. The BSA-Au NCs were freeze-dried and stored at $4^{\circ} \mathrm{C}$ and did not precipitate, even 3 months after re-dispersion in water. The use of the BSA-template-mediated green synthesis route for the production of fluorescent BSA-Au NCs through the proposed mechanism [41] considerably reduced the synthesis time and eliminated the need for further purification of toxic reagents.

\section{Rifampicin detection procedure}

Stock solutions $(10 \mathrm{mM})$ of isoniazid (INZ), rifampicin $(\mathrm{R})$, ethambutol (E) and pyrazinamide (P) were prepared. Subsequently, $1 \mathrm{~mL}$ of the prepared BSA-Au NCs and $100 \mu \mathrm{L}$ of the antibiotic solution were added to a bottle, and the volume was adjusted to $10 \mathrm{~mL}$ to a final antibiotic concentration of $100 \mu \mathrm{M}$. The fluorescence intensities were recorded immediately with an excitation wavelength of $480 \mathrm{~nm}$ and emission wavelength of $640 \mathrm{~nm}$. The calibration curve for rifampicin was prepared according to the normalized decrease in fluorescence intensity, which is defined as $\left(\mathrm{F}_{0}-\mathrm{F}\right) / \mathrm{F}$, where $\mathrm{F}_{0}$ and $\mathrm{F}$ are the maximum emission intensities of the BSA$\mathrm{Au}$ NC system in the absence and presence of rifampicin, respectively.

\section{Characterization of BSA-Au NCs}

Field emission transmission electron microscopy was used on the prepared BSA-Au NCs to calculate the size distribution at $200 \mathrm{kV}$. The samples were prepared by applying the diluted solution (20-fold dilution from the prepared solution) onto formvar stabilized with a carbon
TEM grid (200 mesh size; TED PELLA) followed by drying at room temperature. The MALDI-MS analysis was used to characterize the BSA conjugation with Au atoms. The ICP-MS analysis was applied to determine the Au content of the BSA-Au NCs both in the presence and absence of rifampicin.

\section{BSA-Au NCs on paper platform synthesis}

Advantec chromatography paper sheets (grade No.1, Toyo Roshi Kaisha Ltd) were cut into standard A4 sheets that directly fit into the feed tray of a commercial solid ink printer (ColorQube ${ }^{\mathrm{m}} 8570$, Xerox Corporation). The ink printers were capable of printing patterns that serve as hydrophobic barriers after proper heating. Paper mircroplates were designed with a standard 96-well format. All of the measurements followed the "Corning Microplate Selection guide" (Corning Inc., USA). The printed pattern of a 96-well plate was placed on a hot plate (Corning PC-420d, Corning Inc.) at $150^{\circ} \mathrm{C}$ for $2 \mathrm{~min}[12$, 13], which melted the wax and created a hydrophobic barrier because of vertical percolation of the wax in the paper. Each well was imbued with $30 \mu \mathrm{L}$ of the prepared BSA-Au NCs and allowed to dry at $40^{\circ} \mathrm{C}$ for $30 \mathrm{~min}$. The microplates were stored in the dark at room temperature $\left(25-35^{\circ} \mathrm{C}\right)$.

\section{Additional file}

Additional file 1: Supplementary Data.

\section{Abbreviations}

BSA: bovine serum albumin; TB: tuberculosis; R: rifampicin; INZ: isoniazid; E: ethambutol; P: pyrazinamide; BSA-Au NCs: bovine serum albumin-stabilized gold nanoclusters; NCs: nanoclusters.

\section{Authors' contribution}

PC supervised the study and contributed to the writing of this article, selection of the methodology, and finalization of the manuscript. KC designed and executed all of the experimental studies, analyzed the data, and drafted the manuscript. CWK was involved in the preparation of the manuscript. AC procured urine from healthy subjects and supervised the regulations associated with the study. All authors have read and approved the final manuscript.

\section{Author details \\ ${ }^{1}$ Department of Engineering and System Science, National Tsing Hua University, Hsinchu 300, Taiwan. ${ }^{2}$ Nano Science and Technology Program, Taiwan International Graduate Program, Academia Sinica, Taipei 115, Taiwan. ${ }^{3}$ National Tsing Hua University, Hsinchu 300, Taiwan. ${ }^{4}$ Research Center for Applied Sciences, Academia Sinica, Taipei 115, Taiwan, ROC. ${ }^{5}$ Department of Pathology, Tri-Service General Hospital, National Defense Medical Center, Taipei, Taiwan.}

\section{Acknowledgements}

This study was supported by the Ministry of Science and Technology (MOST) of Taiwan (contracts MOST 103-2113-M-001-008-MY3) and Academia Sinica Research Project on Nano Science and Technology. CWK acknowledges financial support received from the MOST via funding award NSC-102-2113-M-001019-MY3. The authors thanks Mr. Abhishek Pathak for providing assistance in obtaining the FTIR images and Ms. Feby Wijaya Pratiwi and Ms. Sreerupa Sarkar for providing valuable input that improved the quality of the manuscript. 


\section{Compliance with ethical guidelines}

\section{Competing interests}

The authors declare that they have no competing interests.

Received: 9 February 2015 Accepted: 17 June 2015

Published online: 26 June 2015

\section{References}

1. Khalili H, Dasthi-khavidaki S, Sajadi S, Hajiabolbaghi M (2008) Assessment of adherence to tuberculosis drug regimen. DARU J Pharm Sci 16:1

2. Fraimow HS (2009) Antimicrobial therapy in osteomyelitis. Semin Plast Surg 23(2):90-99

3. Forrest GN, Tamura K (2010) Rifampin combination therapy for nonmycobacterial infections. Clin Microbiol Rev 23:14-34

4. Mansilla AE, Valenzuela MIA, de la Pena M, Salinas F, Canada FC (2001) Comparative study of partial least squares and a modification of hybrid linear analysis calibration in the simultaneous spectrophotometric determination of rifampicin, pyrazinamide and isoniazid. Anal Chim Acta 427:129-136

5. Halvatzis SA, Timotheou-Potamia MM, Hadjiioannou TP (1993) Continuous-flow chemiluminometric determination of dihydralazine, rifampicin and rifamycin SV by oxidation with $\mathrm{N}$-bromosuccinimide. Anal Chim Acta 272:251-263

6. Lomillo MAA, Renedo OD, Martinez JMA (2001) Resolution of ternary mixtures of rifampicin, isoniazid and pyrazinamide by differential pulse polarography and partial least squares method. Anal Chim Acta 449:167-177

7. Swart KJ, Papgis M (1992) Automated high-performance liquid chromatographic method for the determination of rifampicin in plasma. J Chromatogr A 593(1-2):21-24

8. Panchagnula R, Sood A, Sharda N, Kaur K, Kaul CL (1999) Determination of rifampicin and its main metabolite in plasma and urine in presence of pyrazinamide and isoniazid by HPLC method. J Pharm Biomed Anal 18(6):1013-1020

9. Calleja I, Blanco-Príeto MJ, Ruz N, Renedo MJ, Dios-Viéitez MC (2004) High-performance liquid-chromatographic determination of rifampicin in plasma and tissues. J Chromatogr A 1031:289-294

10. Riva E, Merati R, Cavenaghi L (1992) High-performance liquid chromatographic determination of rifapentine and its metabolite in human plasma by direct injection into a shielded hydrophobic phase column. J Chromatogr A 553:35-40

11. Calleri E, De Lorenzi E, Furlanetto S, Massolini G, Caccialanza G (2002) Validation of a RP-LC method for the simultaneous determination of isoniazid, pyrazinamide and rifampicin in a pharmaceutical formulation. J Pharm Biomed Anal 29:1089-1096

12. Carrilho E, Martinez AW, Whitesides GM (2009) Understanding wax printing: a simple micropatterning process for paper-based microfluidics. Anal Chem 81:7091-7095

13. Cheng CM, Martinez AW, Gong J, Mace CR, Phillips ST, Carrilho E et al (2010) Paper-based ELISA. Angew Chem 49:4771-4774

14. Tsou CJ, Hsia CH, Chu JY, Hung Y, Chen YP, Chien FC et al (2015) Local pH tracking in living cells. Nanoscale 7:4217-4225

15. Ruedas-Rama MJ, Walters JD, Orte A, Hall EAH (2012) Fluorescent nanoparticles for intracellular sensing: a review. Anal Chim Acta 751:1-23

16. Saha K, Agasti SS, Kim C, Li X, Rotello VM (2012) Gold nanoparticles in chemical and biological sensing. Chem Rev 112:2739-2779

17. Charan S, Chien FC, Singh N, Kuo CW, Chen P (2011) Development of lipid targeting Raman probes for in vivo imaging of C. elegans. Chem Eur J 17:5165-5170

18. Segev-Bar M, Haick H (2013) Flexible sensors based on nanoparticles. ACS Nano 7:8366-8378

19. Singh N, Charan S, Sanjiv K, Huang SH, Hsiao YC, Kuo CW et al (2012) Synthesis of tunable and multifunctional Ni-doped near-infrared QDs for cancer cell targeting and cellular sorting. Bioconj Chem 23:421-430

20. Xie J, Zheng Y, Ying JY (2009) Protein-directed synthesis of highly fluorescent gold nanoclusters. JACS 131:888-889

21. Wei H, Wang Z, Zhang J, House S, Gao YG, Yang L et al (2011) Timedependent, protein-directed growth of gold nanoparticles within a single crystal of lysozyme. Nat Nanotechnol 6:93-97
22. Mayavan S, Dutta NK, Choudhury NR, Kim M, Elvin CM, Hill AJ (2011) Selforganization, interfacial interaction and photophysical properties of gold nanoparticle complexes derived from resilin-mimetic fluorescent protein rec1-resilin. Biomaterials 32:2786-2796

23. Gúevel XL, Daum N, Schneider M (2011) Synthesis and characterization of human transferrin-stabilized gold nanoclusters. Nanotechnology $22: 275103$ (7p)

24. Shichibu Y, Negishi Y, Tsunoyama H, Kanehara M, Teranishi T, Tsukuda T (2007) Extremely high stability of glutathionate-protected $\mathrm{Au}_{25}$ clusters against core etching. Small 3(5):835-839

25. Garcia AR, Rahn I, Johnson S, Patel R, Guo J, Orbulescu J et al (2013) Human insulin fibril-assisted synthesis of fluorescent gold nanoclusters in alkaline media under physiological temperature. Colloids Surf B 105:167-172

26. Wen F, Dong Y, Feng L, Wang S, Zhang S, Zhang X (2011) Horseradish peroxidase functionalized fluorescent gold nanoclusters for hydrogen peroxide sensing. Anal Chem 83:1193-1196

27. Chen Y, Wang Y, Wang C, Li W, Zhou H, Jiao H et al (2013) Papain-directed synthesis of luminescent gold nanoclusters and the sensitive detection of $\mathrm{Cu}^{2+}$. J Colloid Interface Sci 396:63-68

28. Chen Z, Qian S, Chen X, Gao W, Lin Y (2012) Protein-templated gold nanoclusters as fluorescence probes for the detection of methotrexate. Analyst 137:4356-4361

29. Chen Z, Qian S, Chen J, Chen X (2012) Highly fluorescent gold nanoclusters based sensor for detection of quercetin. J Nanopart Res 14:1264

30. Liu Y, Ai K, Cheng X, Huo L, Lu L (2010) Gold-nanocluster-based fluorescent sensors for highly sensitive and selective detection of cyanide in water. Adv Func Mater 20:951-956

31. Xia X, Long Y, Wang J (2013) Glucose oxidase-functionalized fluorescent gold nanoclusters as probes for glucose. Anal Chim Acta 772:81-86

32. Wei H, Wang Z, Yang L, Tian S, Hou C, Lua Y (2010) Lysozyme-stabilized gold fluorescent cluster: synthesis and application as $\mathrm{Hg}^{2+}$ sensor. Analyst 135(6):1406-1410

33. Lin Z, Luo F, Dong T, Zheng L, Wang Y, Chi Y et al (2012) Recyclable fluorescent gold nanocluster membrane for visual sensing of copper (II) ion in aqueous solution. Analyst 137:2394-2399

34. Durgadas CV, Sharma CP, Sreenivasan K (2011) Fluorescent gold clusters as nanosensors for copper ions in live cells. Analyst 136:933-940

35. Liu X, Zong C, Lu L (2012) Fluorescent silver nanoclusters for user-friendly detection of $\mathrm{Cu}^{2+}$ on a paper platform. Analyst 137:2406-2414

36. Yu YO, Cheng YF, Huang SY, Bai AM, Hu YJ (2011) Probing the binding of rifampicin to bovine serum albumin in aqueous solution. J Solut Chem 40:1711-1723

37. Markarian SA, Aznauryan MG (2012) Study on the interaction between isoniazid and bovine serum albumin by fluorescence spectroscopy: the effect of dimethylsulfoxide. Mol Biol Rep 39:7559-7567

38. Martinez AW, Phillips ST, Carrilho E, Thomas III SW, Sindi H, Whitesides GM (2008) Simple telemedicine for developing regions: camera phones and paper-based microfluidic devices for real-time, off-site diagnosis. Anal Chem 80:3699-3707

39. Veigas B, Jacob JM, Costa MC, Santos DS, Inácio MVJ, Martins R et al (2012) Gold on paper-paper platform for Au-nanoprobe TB detection. Lab on a Chip 12:4802-4808

40. Iqbal Z, Eriksson M (2013) Classification and quantitative optical analysis of liquid and solid samples using a mobile phone as illumination source and detector. Sens Actuators B Chem 185:354-362

41. Chen PC, Chiang CK, Chang HT (2013) Synthesis of fluorescent BSA-Au NCs for the detection of $\mathrm{Hg}^{2+}$ ions. J Nanopart Res 15:1336

42. Turel I, Bukovec P, Quirds M (1997) Crystal structure of ciprofloxacin hexahydrate and its characterization. Int J Pharm 152:59-65

43. Kerns EH, Rourick RA, Volk KJ, Lee MS (1997) Buspirone metabolite structure profile using a standard liquid chromatographic-mass spectrometric protocol. J Chrom B 698:133-145

44. Mokrosz JL, Pietrasiewicz M, Duszyiiska B, Cegla MT (1992) Structureactivity relationship studies of central nervous system agents. 5. Effect of the hydrocarbon chain on the affinity of 4-substituted 1-(3-Chlorophenyl) piperazines for 5-HT1A receptor site. J Med Chem 35:2369-2374

45. Gurumurthy P, Ramachandran G, Kumar AKH, Rajasekaran S, Padmapriyadarsini C, Swaminathan S et al (2004) Decreased bioavailability of rifampin and other antituberculosis drugs in patients with advanced human immunodeficiency virus disease. Antimicrob Agents Chemother 48:4473-4475 